\title{
On the BRIP Algorithms Security for RSA
}

\author{
Frédéric Amiel $^{1}$ and Benoit Feix ${ }^{2}$ \\ 1 AMESYS, \\ 1030, Avenue Guillibert de la Lauzire, \\ 13794 Aix-en-Provence, Cedex 3, France \\ f.amiel@amesys.fr \\ ${ }^{2}$ INSIDE CONTACTLESS \\ 41 Parc Club du Golf \\ 13856 Aix-en-Provence, Cedex 3, France \\ bfeix@insidefr.com
}

\begin{abstract}
Power Analysis has been intensively studied since the first publications in 1996 and many related attacks on naive implementations have been proposed. Nowadays algorithms in tamper resistant devices are protected by different countermeasures most often based on data randomization such as the BRIP algorithm on ECC and its RSA derivative. However not all of them are really secure or in the best case proven to be secure. In 2005, Yen, Lien, Moon and Ha introduced theoretical power attacks on some classical and BRIP exponentiation implementations, characterized by the use of a chosen input message value \pm 1 . The first part of our article presents an optimized implementation for BRIP that takes advantage of the Montgomery modular arithmetic to speed up the mask inversion operation. An extension of the Yen et al. attack, based on collision detection through power analysis, is also presented. Based on this analysis we give security advice on this countermeasure implementation and determine the minimal random length to reach an appropriate level of security.
\end{abstract}

Keywords: Power analysis, collision attacks, RSA, BRIP, modular multiplication and exponentiation.

\section{Introduction}

Asymmetric cryptography was introduced by Diffie and Hellman [DH76] in 1976. The most widely used algorithms today are: RSA [RSA78] invented in 1978 by Rivest, Shamir, and Adleman, and elliptic curve cryptosystems (ECC) independently introduced by Koblitz [Kob87] and Miller [Mil86].

Compared with symmetric cryptography, public key algorithms are computationally very intensive. In practice long integer arithmetic is most often handled by specific coprocessors designed for efficient computation in $G F(p)$. This is the case for embedded solutions with strict power consumption and/or timing constraints.

Initially smart cards were considered inherently tamper resistant because any private data was embedded and thus physically inaccessible to an unauthorized

(C) IFIP International Federation for Information Processing 2008 
user. However in 1996 timing attacks were publicly introduced by Kocher in [Koc96]. Two years later he also introduced power analysis attacks with Jaffe and Jun [KJJ99]. Side Channel Analysis (SCA) is a group of techniques including simple power analysis (SPA) and differential power analysis (DPA). SCA threatens any naive cryptographic algorithm implementation. Since these first articles were published, power analysis has been widely investigated, some publications have focused on countermeasures and their drawbacks [FV03, MPO05, YLMH05] whereas others have focused on improving the efficiency of the attacks [ABDM00, BK03, BCO04].

One such countermeasure is the Binary with Random Initial Point (BRIP) algorithm(s) by Mamiya, Miyaji, Morimoto [MMM04] and Itoh, Izu and Takenada [IIT04], later improved in [IIT06]. BRIP countermeasure was originally designed for ECC and later extended to RSA cryptosystems. Its RSA variant corresponds to the countermeasure also proposed in $\left[\mathrm{KHK}^{+} 04\right]$ and is particularly interesting in terms of implementation as neither the bit size of the prime characteristic of the field is increased nor is the knowledge of the public exponent value needed.

Our study focuses on the exponentiation and for readibility purposes, BRIP acronym will refer here to the BRIP RSA derivative of the countermeasure.

The paper is organized as follows: section 2 gives an overview of embedded asymmetric algorithms and their related side-channel potential vulnerabilities. Section 3 describes the BRIP algorithms with the current identified vulnerabilities and our implementation improvements. New attacks on these algorithms and recommendations will be presented in Section 4. We conclude our research in Section 5 .

\section{Power Analysis Background}

Since the initial publication in [KJJ99] on Simple Power Analysis (SPA), many improvements have been made on this subject. Electronic devices, such as smart cards or other security products, are designed with thousands of logical gates switching differently depending on the executed operations and the data manipulated. The device power consumption of the chip depends on these operations which can be easily monitored and analysed on an oscilloscope. For instance, if the square operation has a different pattern on the power curve than the one for multiplication, it is obvious that the attacker can easily recover the secret exponent in a naive RSA implementation. Many other differences visible in the power curve can lead to the same kind of leakage on the private key(s). Developers must take into account all the potential vulnerabilities.

One of the first Collision Power Analysis attack is the Doubling Attack by Fouque and Valette [FV03]. It was applied on a scalar multiplication operation in ECC. They also explained how it could be extended to RSA implementations.

Differential Power Analysis (DPA) and its improvements represent the other main class of side channel attacks. The most well known is the Correlation Power Analysis (CPA) by Brier, Clavier and Olivier [BCO04]. It was later applied by Amiel, Feix and Villegas [AFV07] on most asymmetric algorithms. The first 
DPA attack on RSA was done in 1999 by Messerges, Dabbish and Sloan [MDS99]. Enhanced DPA attacks, such as the Zero Value Point Attack published by Goubin in [Gou03], have also been done on elliptic curve implementations. Goubin's attack threatens Coron's randomization of the projective coordinates [Cor99] in the elliptic curve scalar multiplication. The first combination between fault injection and power analysis has also been applied to XTR in [CG04]. The BRIP algorithms have then been proposed to counteract the Zero Value Point Attack. Moreover, BRIP can also be applied in $G F(p)$ for cryptosystems based on the factorization and discrete logarithm problems, like RSA.

However Yen, Lien, Moon and Ha [YLMH05] presented a power collision attack on the BRIP countermeasure for RSA by using \pm 1 values for input messages, and on the Square and Multiply Always algorithm by using $\pm m \bmod n$ messages as an input for RSA.

\section{Modular Exponentiations for BRIP Algorithms}

Firstly we present the BRIP algorithm variant for RSA, we also introduce some improvements and optimizations for this countermeasure when combined with Montgomery modular multiplication.

\subsection{Modular Multiplication and Exponentiation}

We summarize the principles used later in this paper: modular multiplication and exponentiation, in particular the ones designed by Montgomery, which are particularly suitable for embedded implementations and the RSA public key cryptosystem.

\subsection{Modular Multiplication}

To compute modular multiplications $x \times y \bmod n$ on long integers $x, y$ and $n$ Montgomery proposed the following efficient algorithm in [Mon85].

\section{Montgomery Modular Multiplication}

Given a modulus $n$ and two integers $x$ and $y$, of size $v$ in base $b$, with $\operatorname{gcd}(n, b)=1$ and $r=b^{\left\lceil\log _{b}(n)\right\rceil}$, MontMul algorithm computes:

$$
\operatorname{MontMul}(x, y, n)=x \times y \times r^{-1} \bmod n
$$

We suggest the reader to refer to Appendix A.1 and papers [Mon85] and [KAK96] if more detail on this operation is wished.

We can then use this operation to process efficiently Montgomery modular exponentiation (MontExp) as detailed in [Dhe98]. Compared to a classical Square and Multiply algorithm it consists of multiplying the message operand and the accumulator by $r \bmod n$ before the exponentiation loop. In this case any intermediate result during the exponentiation is equal to $m^{k} \cdot r \bmod n$. At the end 
the $r$ value is removed by doing a modular montgomery multiplication by 1 . Refer to Appendix A.2 for the detailed algorithm.

\section{Classical BRIP Implementation for RSA}

Alg. 3.1 describes the classical BRIP implementation introduced in $\left[\mathrm{KHK}^{+} 04\right]$ with a random $v$ generated from a $h$-bit random seed $u$. It means $v=f(u)$. Value $v$ must be as long as the modulus to prevent the implementation of a chosen message SPA. The security of the random value $v$ is the same as the seed random $u$, this implies there are only $2^{h}$ possible values. For instance $v=(u|u \ldots| u)$.

The major drawback is the time needed to compute the modulo inverse $v^{-1}$ $\bmod n$. The next implementation avoids this if Montgomery modular multiplication hardware is available.

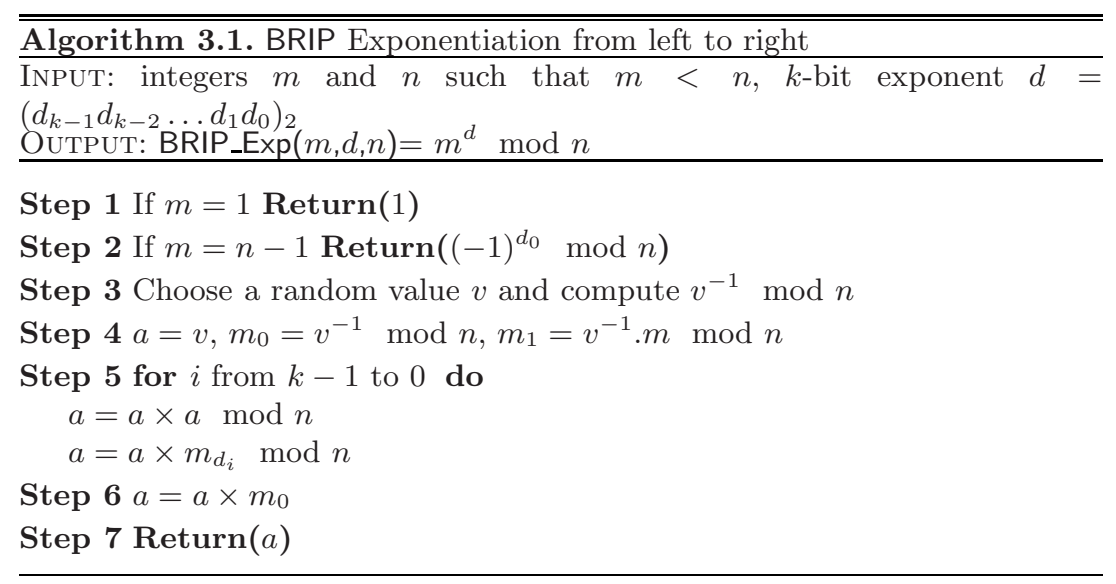

\section{Second BRIP Implementation with MontMul}

The inversion of random $v \bmod n$ is a penalty for the BRIP algorithm performance. A solution consists in using the following property of the Montgomery multiplication: $\operatorname{Mont} \operatorname{Mul}(1,1, n)=r^{-1} \bmod n$. This gives an efficient way to compute an exponentiation with both a fixed base value $(r)$ and a negative exponent. The idea is presented by Ciet and Feix in [CF05] and can also be applied to BRIP.

The $v^{-1} \bmod n$ computation can be replaced by $r^{-v} \bmod n$ implemented as an exponentiation with a relatively short exponent (typically $|v|<<|d|$ ). This trick saves a lot of time compared to a modular inverse calculation.

Thus we obtained the Algorithm Alg. 3.2.

Step 5. of Alg. 3.2 replaces the costly inversion operation of random $v$ in Alg. 3.1. However both previous algorithms Alg. 3.1 and Alg. 3.2 have a complexity of 2 which is the same as the well known Square and Multiply Always algorithm. Improvements can however be envisaged by using $k$-ary and sliding window methods [CKK]. In [MMM04] the authors also presented optimized versions of $\mathrm{BRIP}$, one version is using the $k$-ary method. 

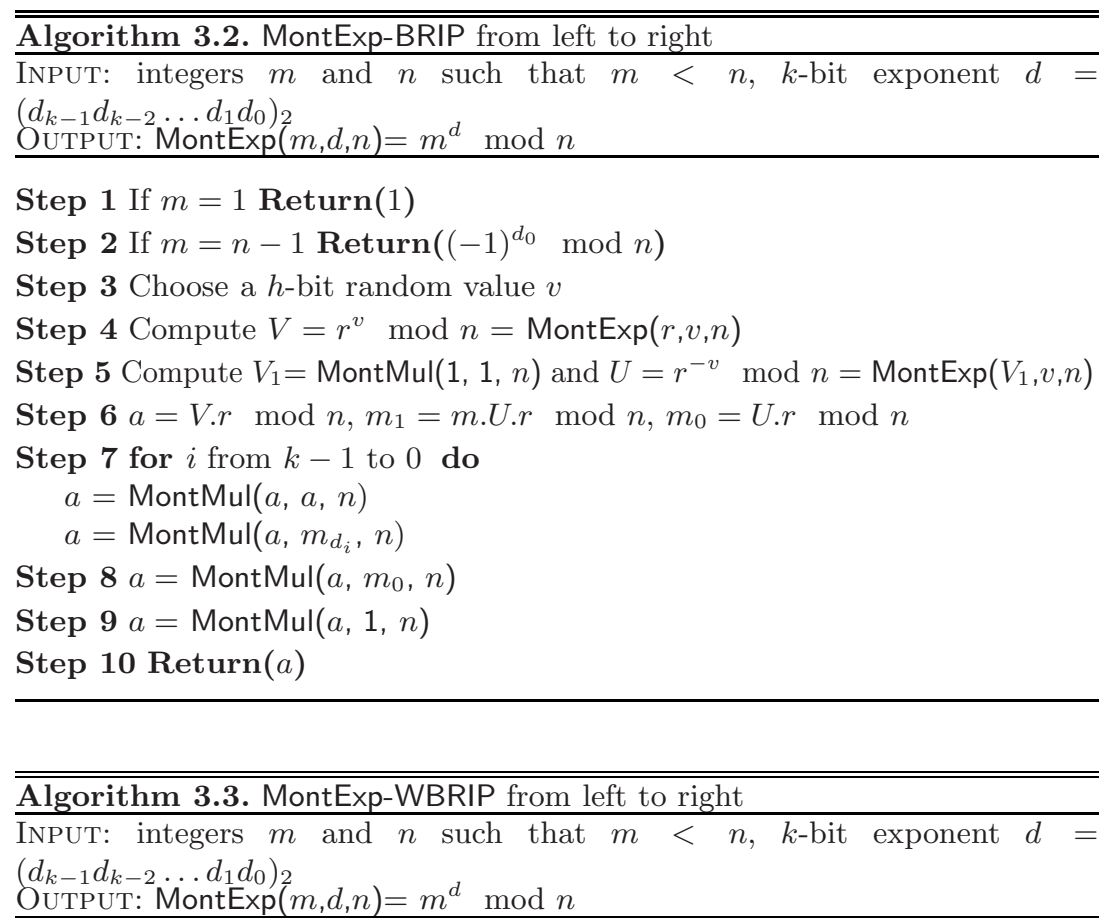

Step 1 If $m=1$ Return(1)

Step 2 If $m=n-1 \operatorname{Return}\left((-1)^{d_{0}} \bmod n\right)$

Step 3 Choose a $h$-bit random value $v$

Step 4 Compute $V=r^{v} \bmod n=\operatorname{MontExp}(r, v, n)$

Step 5 Compute $U=r^{-3 v} \bmod n$

Step 6 Compute $a=V \cdot r \bmod n, m_{0}=U . r \bmod n, m_{1}=$ m.U.r $\bmod n$

Step 7 Compute $m_{2}=m^{2} \cdot U \cdot r \bmod n, m_{3}=m^{3} \cdot U \cdot r \bmod n$

Step 8 for $i$ from $k-1$ to 0 by 2 do

$a=\operatorname{MontMul}(a, a, n)$

$a=\operatorname{MontMul}(a, a, n)$

$a=\operatorname{MontMul}\left(a, m_{\left(2 . d_{i}+d_{i-1}\right)}, n\right)$

Step $9 a=\operatorname{MontMul}\left(a, m_{0}, n\right)$

Step $10 a=\operatorname{MontMul}(a, 1, n)$

Step 11 Return $(a)$

Algorithm Alg. 3.3 we present here, corresponds to WBRIP for RSA with MontMul. It corresponds to a 2-ary exponentiation with the BRIP countermeasure and the improvement we proposed with the Montgomery multiplication. There is no costly inversion operation and the algorithm complexity is 1.5 , but more memory space is required for the pre-computations storage compared to both the previous versions. 
In this case the mask value for computation is no longer $r^{-v}$ but $r^{-3 v}$ as we manipulate scalar bits by 2-bit windows.

Depending on the memory contraints, the size of the window can be modified. For a $k$-bit window the algorithm complexity becomes equal to $1+1 / k$.

\section{Power Analysis Attacks on BRIP Like Algorithms}

We present here an improvement to the power collision attack on RSA implementations based on the previous BRIP implementations. Fouque and Valette first [FV03] introduced power collision attacks on some of the classical elliptic curve scalar multiplication algorithms, they also explained how to extend the technique to modular exponentiations. Later Yen et al. [YLMH05] introduced collision power attacks based on chosen message values $\pm 1 \bmod n$ that allows the secret exponent value $d$ to be recovered from a single curve. Developpers must avoid BRIP computation when the input message equals $n-1$ and simply return value 1 or $n-1$ depending on the parity of the secret exponent.

In their article, some other variants of the attacks are presented, especially on the Square and Multiply Always algorithm by using $\pm m \bmod n \operatorname{messages}$ as input, but none of them compromise a full implementation of BRIP.

\subsection{Collision Power Analysis on BRIP and MontExp-BRIP}

Modular multiplication on a chip requires relatively long processing time and relatively high power consumption compared with symmetric algorithms, where for example, processing can be carried out in a few clock cycles in hardware implementations of AES.

In figure 1 we analyse power traces of the MonMult operation executed on a tamper resistant device such as a smart card.

We choose two different random messages $m_{1}$ and $m_{2}$ and for each message we execute three multiplications $\operatorname{MontMul}\left(m_{1}, m_{1}, n\right)$ and $\operatorname{MontMul}\left(m_{2}, m_{2}, n\right)$. We then collect the three power curves $C_{1,1}, C_{1,2}$ and $C_{1,3}$ of the multiplication with $m_{1}$ and three curves $C_{2,1}, C_{2,2}$ and $C_{2,3}$ of the multiplication with $m_{2}$.

We notice, cf. figure 1 , that on the selected chip, the multiplication is a very power consuming operation. This is due to the large number of gates which are switching together in the asymmetric coprocessor logic.

From this curves we observe that power collisions occur for similar data manipulated by the chip. $C_{1,1}, C_{1,2}$ and $C_{1,3}$ are similar and have exactly the same power traces, as do $C_{2,1}, C_{2,2}$ and $C_{2,3}$. It means that $C_{i, j}$ collides with $C_{k, l}$ when $i=k$ while $C_{i, j}$ is different from $C_{k, l}$ when $i \neq k$.

Due to the important number of clock cycles in a modular multiplication in the power curve, we can assume that different input data will have different power trace patterns. This means we can distinguish collisions with a high probability. The tests we made on the selected chip confirm our assumption.

We analyse if we can exploit eventual collisions on the classical BRIP algorithm 3.1 and the MontExp-BRIP 3.2 for an $h$-bit random value $v$. For both algorithms the analysis will be identical. 


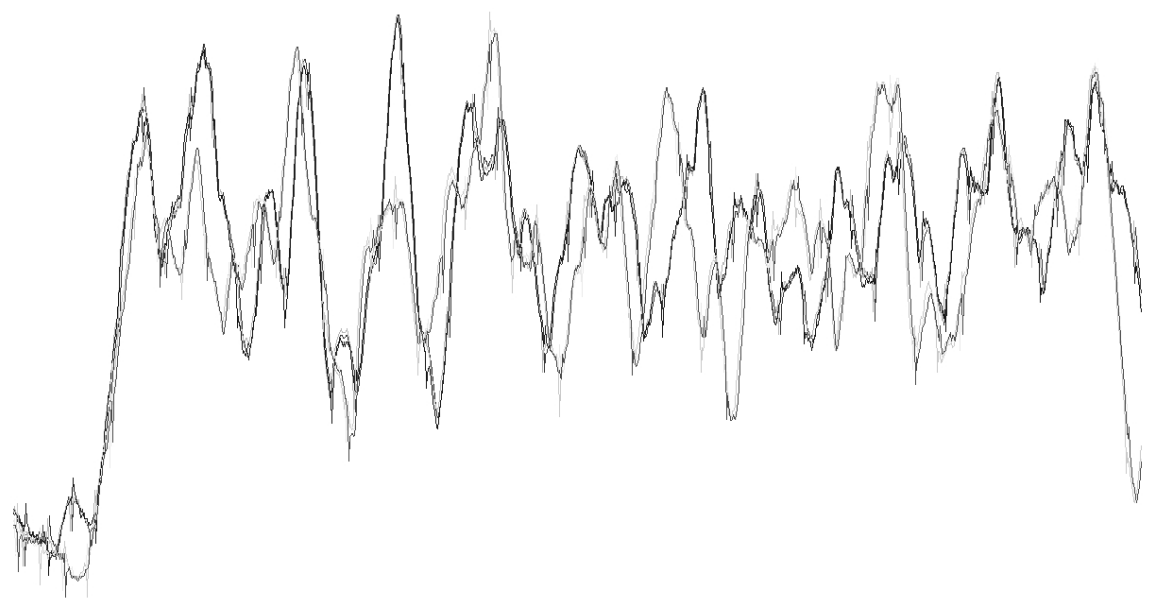

Fig. 1. Power consumption of a single modular multiplication for curves $C_{1,1}, C_{1,2}$, $C_{1,3}$ and $C_{2,1}, C_{2,2}, C_{2,3}$

Depending on the method for generating the random value $v$, it is obvious that in some cases, collisions on its values could happen when generating it. This will depend on the quality of the random and on its length. However for performance reasons BRIP and especially MontExp-BRIP can not use big values $h$.

We observe that if a colliding value for the random mask $v$ appears, then by choosing as algorithm input message $m$ for the first execution and $-m \bmod n$ for the second one, we can have multiplications with similar operands in both executions. This could lead to distinguishable power collisions between the power curves of both executions. We then try to exploit these collisions to recover the secret exponent $d$.

Firstly we execute BRIP a number of times with input message $m$. For any execution a new random value $v_{1}$ is generated by the chip. Then we repeat this operation with the input message $-m \bmod n$, for each execution a new random value $v_{2}$ is generated.

Let $d=d^{\prime \prime} \cdot 2^{i+1}+d_{i} \cdot 2^{i}+d^{\prime}$ where, $d_{i}$ is the current bit handled by the exponentiation loop, $d^{\prime \prime}$ the left part of $d$ previously processed (left-to-right exponentiation) and $d^{\prime}$ the right remaining part of the exponent. In figure 2 we can observe for a step $i$ of the BRIP execution what operands are manipulated by the chip for modular multiplications. In the first table we see these operand values during a real multiplication $\left(d_{i}=1\right)$, and in the second table when $d_{i}=0$.

We detect collisions on any Fake Multiplication (multiplication by $r^{-v}$ ) operation when a collision happens on $v$ and $v_{1}=v_{2}$. Thus collision detection through power analysis is a real threat.

We can then observe on power traces when a collision occurs. We store in memory the power curves $C_{i}$ of the BRIP execution with message $m$ and $C_{i}^{\prime}$ with message $-m \bmod n$. Then we search for two curves $C_{i}$ and $C_{j}^{\prime}$ where 


\begin{tabular}{|c||c|c|c||}
\hline Message & Square & Message Multiplication $\left(d_{i}=1\right)$ & Square \\
\hline \hline$m$ & {$\left[m^{d^{\prime \prime}} \cdot v_{1}\right]^{2}$} & {$\left[\left(m^{2 \cdot d^{\prime \prime}}\right) \cdot v_{1}^{2}\right] \times\left[m \cdot v_{1}^{-1}\right]$} & {$\left[\left(m^{2 \cdot d^{\prime \prime}+1}\right) \cdot v_{1}\right]^{2}$} \\
$-m$ & {$\left[(-m)^{d^{\prime \prime}} \cdot v_{2}\right]^{2}$} & $\left.\left[\left(m^{2 \cdot d^{\prime \prime}}\right) \cdot v_{2}^{2}\right)\right] \times\left[-m \cdot v_{2}^{-1}\right]$ & {$\left[\left((-m)^{2 \cdot d^{\prime \prime}+1}\right) \cdot v_{2}\right]^{2}$} \\
\hline Collision if $v_{1}=v_{2}$ & - & No & No \\
\hline \hline Message & Square & Fake Multiplication $\left(d_{i}=0\right)$ & Square \\
\hline$m$ & {$\left[m^{d^{\prime \prime}} \cdot v_{1}\right]^{2}$} & {$\left[\left(m^{2 \cdot d^{\prime \prime}}\right) \cdot v_{1}^{2}\right] \times\left[v_{1}^{-1}\right]$} & {$\left[\left(m^{2 \cdot d^{\prime \prime}}\right) \cdot v_{1}\right]^{2}$} \\
$-m$ & {$\left[(-m)^{d^{\prime \prime}} \cdot v_{2}\right]^{2}$} & $\left.\left[\left(m^{2 \cdot d^{\prime \prime}}\right) \cdot v_{2}^{2}\right)\right] \times\left[v_{2}^{-1}\right]$ & {$\left[\left(m^{2 \cdot d^{\prime \prime}}\right) \cdot v_{2}\right]^{2}$} \\
\hline Collision if $v_{1}=v_{2}$ & - & Yes & Yes \\
\hline
\end{tabular}

Fig. 2. BRIP execution for $d_{i}=1$ and $d_{i}=0$

\begin{tabular}{l}
\hline \hline Algorithm 4.4. BRIP Collision Attack \\
InPUT: $s=\operatorname{RSA-BRIP}(m, d), s^{\prime}=\operatorname{RSA-BRIP}(-m, d)$ \\
OUtPUT: Secret exponent $d$
\end{tabular}

Step 1 Choose a random value $m$ in $[2, n-2]$.

Step 2 Collect $k$ traces $\left(C_{0}, \ldots, C_{k-1}\right)$ of BRIP execution with $m$ as input message.

Step 3 Collect $k$ traces $\left(C_{0}^{\prime}, \ldots, C_{k-1}^{\prime}\right)$ of BRIP execution with $-m$ as input message.

Step 4 Find traces $C_{i}$ and $C_{j}^{\prime}$ such as both traces are colliding on each BRIP Fake Multiply.

Step 5 Compute $S=\left|C_{i}-C_{j}^{\prime}\right|$.

Step 6 Each non zero difference on $S$ identify a true multiplication, i.e. $d_{i}=1$

power collisions appear between the two curves. Then by subtracting $C_{j}^{\prime}$ to $C_{i}$ we can recover the secret exponent $d$.

The probability of finding at least one colliding couple from both sets of $k$ traces is approximated in [MOV96] (Fact 2.27) by:

$$
p_{\text {collision }} \simeq 1-e^{-\left(\left(k^{2}\right) /|h|\right)}
$$

where $|h|$ denotes the number of possible value for $v$ so $2^{h}$.

Figure 3 gives the probability of collision for a 32-bit random $v$ relative to the number of encryptions done.

Thus in practice with $2^{32}$ possible values for $v$ (32-bit random), two sets of $k=78000$ curves are sufficient to have a probability of $\frac{1}{2}$ for obtaining a collision, where two sets of $k=200000$ curves will lead to a collision and then a successful attack in 99 percents of cases. $\left(p_{\text {collision }} \simeq 0.99\right)$.

This collision attack is a serious threat and also appears on MontExp-BRIP, cf. Alg. 3.2. The random value $v$ is not used in the same way in the algorithm but the analysis and the results of collision are similar.

We suggest using at least 96-bit random value $v(h=96 \mathrm{cf}$. figure 5$)$ to prevent MontExp-BRIP and BRIP against such collision attacks. However it is obvious 


\begin{tabular}{|c|c|c|}
\hline$h$ & $k$ & collision \\
\hline \hline 32 & 78000 & 0.507 \\
32 & $2^{17} \approx 131072$ & 0.864 \\
32 & 161000 & 0.951 \\
32 & 200000 & 0.990 \\
32 & $2^{18} \approx 262144$ & 0.999 \\
\hline
\end{tabular}

Fig. 3. Probability of collision for $h=32$

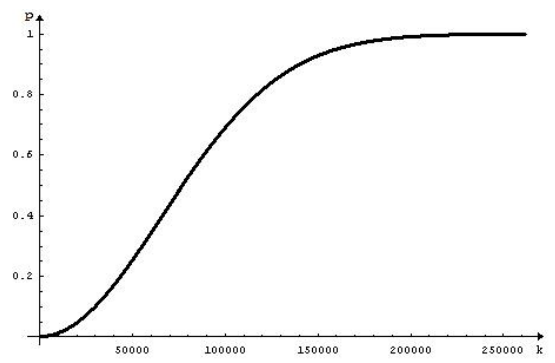

Fig. 4. Graph of probability of collision for a 32-bit value $v$

\begin{tabular}{|c|c|c|}
\hline$h$ & $k$ & collision probability \\
\hline \hline 16 & $2^{9}=512$ & 0.864 \\
16 & $2^{10}=1024$ & 0.999 \\
64 & $5.1 \times 10^{9}$ & 0.505 \\
64 & $2^{33}$ & 0.864 \\
64 & $2^{34}$ & 0.999 \\
96 & $3.3 \times 10^{14}$ & 0.497 \\
96 & $2^{48}$ & 0.864 \\
96 & $2^{49}$ & 0.999 \\
\hline
\end{tabular}

Fig. 5. Probability of collision for other $h$ values

that such random lengths will reduce the performance of these implementations and then the reason for using them.

\subsection{Collision Attack on MontExp-WBRIP}

We analyse the impact of power collisions in the MontExp-WBRIP implementation. In the figure 6 we replace the notation MontMul by MM.

Let $d=d^{\prime \prime} \cdot 2^{i+1}+d_{i} \cdot 2^{i}+d_{i-1} \cdot 2^{i-1}+d^{\prime}$ where $d_{i}$ and $d_{i-1}$ are the two bits of the 2-bit window handled for each exponentiation loop, $d^{\prime \prime}$ the left part of $d$ previously processed and $d^{\prime}$ the right part being process in next steps. 


\begin{tabular}{|c|c|c|c|}
\hline Message & Square & Square & $M_{\left(2 d_{i}+d_{i-1}=0\right)}$ MontMul \\
\hline$m$ & $M M\left(m^{d^{\prime \prime}} \cdot r^{v_{1}}, m^{d^{\prime \prime}} \cdot r^{v_{1}}\right)$ & $M M\left(m^{2 . d^{\prime \prime}} \cdot r^{v_{1}}, m^{2 . d^{\prime \prime}} \cdot r^{v_{1}}\right)$ & $\bar{M} M M\left(m^{4 . d^{\prime \prime}} \cdot r^{4 v_{1}}, r^{-3 v_{1}}\right)$ \\
\hline$-m$ & $M M\left((-m)^{d^{\prime \prime}} \cdot r^{v_{2}},(-m)^{d^{\prime \prime}} \cdot r^{v_{2}}\right)$ & $M M\left(m^{2 \cdot d^{\prime \prime}} \cdot r^{v_{2}}, m^{2 \cdot d^{\prime \prime}} \cdot r^{v_{2}}\right)$ & $M M\left(m^{4 \cdot d^{\prime \prime}} \cdot r^{4 v_{2}}, r^{-3 v_{2}}\right)$ \\
\hline If $v_{1}=v_{2}$ & - & Yes & Yes \\
\hline Message & Square & Square & $M_{\left(2 d_{i}+d_{i-1}=1\right)}$ MontMul \\
\hline$m$ & $M M\left(m^{d^{\prime \prime}} \cdot r^{v_{1}}, m^{d^{\prime \prime}} \cdot r^{v_{1}}\right)$ & $M M\left(m^{2 . d^{\prime \prime}} \cdot r^{v_{1}}, m^{2 . d^{\prime \prime}} \cdot r^{v_{1}}\right)$ & $M M\left(m^{4 \cdot d^{\prime \prime}} \cdot r^{4 v_{1}}, m \cdot r^{-3 v_{1}}\right)$ \\
\hline$-m$ & $M M\left((-m)^{d^{\prime \prime}} \cdot r^{v_{2}},(-m)^{d^{\prime \prime}} \cdot r^{v_{2}}\right)$ & $M M\left(m^{2 \cdot d^{\prime \prime}} \cdot r^{v_{2}}, m^{2 \cdot d^{\prime \prime}} \cdot r^{v_{2}}\right)$ & $M M\left(m^{4 \cdot d^{\prime \prime}} \cdot r^{4 v_{2}},(-m) \cdot r^{-3 v_{2}}\right)$ \\
\hline If $v_{1}=v_{2}$ & - & Yes & No \\
\hline Message & Square & Square & $M_{\left(2 d_{i}+d_{i-1}=2\right)}$ MontMul \\
\hline$m$ & $M M\left(m^{d^{\prime \prime}} \cdot r^{v_{1}}, m^{d^{\prime \prime}} \cdot r^{v_{1}}\right)$ & $M M\left(m^{2 \cdot d^{\prime \prime}} \cdot r^{v_{1}}, m^{2 \cdot d^{\prime \prime}} \cdot r^{v_{1}}\right)$ & $\bar{M} M M\left(m^{4 . d^{\prime \prime}} \cdot r^{4 v_{1}}, m^{2} \cdot r^{-3 v_{1}}\right)$ \\
\hline$-m$ & $M M\left((-m)^{d^{\prime \prime}} \cdot r^{v_{2}},(-m)^{d^{\prime \prime}} \cdot r^{v_{2}}\right)$ & $M M\left(m^{2 . d^{\prime \prime}} \cdot r^{v_{2}}, m^{2 \cdot d^{\prime \prime}} \cdot r^{v_{2}}\right)$ & $M M\left(m^{4 \cdot d^{\prime \prime}} \cdot r^{4 v_{2}}, m^{2} \cdot r^{-3 v_{2}}\right)$ \\
\hline If $v_{1}=v_{2}$ & - & Yes & Yes \\
\hline Message & Square & Square & $M_{\left(2 d_{i}+d_{i-1}=3\right)}$ MontMul \\
\hline$m$ & $M M\left(m^{d^{\prime \prime}} \cdot r^{v_{1}}, m^{d^{\prime \prime}} \cdot r^{v_{1}}\right)$ & $M M\left(m^{2 . d^{\prime \prime}} \cdot r^{v_{1}}, m^{2 \cdot d^{\prime \prime}} \cdot r^{v_{1}}\right)$ & $M M\left(m^{4 \cdot d^{\prime \prime}} \cdot r^{4 v_{1}}, m^{3} \cdot r^{-3 v_{1}}\right)$ \\
\hline$-m$ & $M M\left((-m)^{d^{\prime \prime}} \cdot r^{v_{2}},(-m)^{d^{\prime \prime}} \cdot r^{v_{2}}\right)$ & $M M\left(m^{2 \cdot d^{\prime \prime}} \cdot r^{v_{2}}, m^{2 \cdot d^{\prime \prime}} \cdot r^{v_{2}}\right)$ & $M M\left(m^{4 \cdot d^{\prime \prime}} \cdot r^{4 v_{2}},(-m)^{3} \cdot r^{-3 v_{2}}\right)$ \\
\hline If $v_{1}=v_{2}$ & - & Yes & No \\
\hline
\end{tabular}

Fig. 6. WBRIP execution for possible $2 d_{i}+d_{i-1}$ values

We can observe in figure 6 the different possible collisions on curves when random values $v$ and $v_{1}$ collide. But in WBRIP it does not give us as much information as in the previous algorithms. The collisions will indicate that the 2-bit window value is either 00 or 10 so $d_{i-1}=0$ and non collisions will indicate the 2 -bit window value is either 01 or 11 so $d_{i-1}=1$. Then we recover here half of the bits of the secret exponent $d$.

Indeed, we can extend this result to any $k$-ary implementation of BRIP exponentiation as the Collision Attack gives the information on the parity of $i$ in $m^{i} \cdot r^{-2^{k-1} \cdot v}$ operand used during the Multiplication operation. Therefore the number of bits recovered by a collision is equal to : $|d| / k$, namely $|d|$ for $k=1$, $|d| / 2$ for $k=2$ and so on.

Thus we also suggest using at least 96-bit random value $v$ to prevent MontExpWBRIP from such collision attacks.

\subsection{Collision Attack of BRIP Implementations for RSA CRT}

These collision attacks can be similarly applied to RSA CRT exponentiations protected with MontExp-BRIP, MontExp-WBRIP or BRIP algorithms.

Indeed when $n=p . q, p$ and $q$ being prime numbers of equivalent lengths, choosing $\pm m \bmod n$ messages leads to the manipulation of $\pm m \bmod p$ and $\pm m \bmod q$ in the CRT exponentiations once the reductions by $p$ and $q$ have been done.

Then the previous collision analysis applies identically to RSA CRT using any of the previous BRIP algorithms. 


\subsection{Implementing MontExp-BRIP Countermeasure}

We notice that both exponentiations: $r^{v} \bmod n=\operatorname{MontExp}(r, v, n)$ and $r^{-v}$ $\bmod n=\operatorname{Mont} \operatorname{Exp}\left(V_{1}, v, n\right)$ need to be carefully implemented against the classical power analysis techniques. Indeed it is obvious that if $v$ is recovered, each operand value in algorithm 3.2 becomes deterministic and then statistical attacks can be envisaged to recover the secret exponent.

The most important threat is Timing Attack (TA) for which Double and Add Always or Side Channel Atomicity [CCJ04] are both convenient countermeasures.

Anyway, protection against TA may not be sufficient as the operation $r^{-v}=$ MontExp $\left(V_{1}, v, n\right)$ can be sensitive to SPA. This is due to the particular Hamming Weight of one of the Multiply operands, explicitely $\bar{m}$ in $a=\operatorname{Mon} \operatorname{Mul}(a, \bar{m}, n)$ with $\bar{m}=f_{n}(m)=r^{-1} * r=1$.

Analysing the implementation details of MontMul gives some clues to explaining the leakage. During the computation of $\operatorname{Mont} \operatorname{Mul}(a, 1, n)$, we notice than most of multiplications involved in Step 2 of algorithm A.5 are composed of integer multiplications by 0 or 1 which have a straightforward impact on the power consumption by significantly lowering it compared to the multiplication of two random operands. It can then be feasible to deduce directly from the power curve the nature of each operation and recover $v$ value for each curve.

A simple tweak to counteract such an SPA attack is to compute $(-r)^{-v}$ rather than $r^{-v}, n-1$ will then replace 1 as input operand of MontMult during Multiply operations. This may still not be sufficient to protect against advanced SPA or Template Analysis attacks as intrinsically $r^{-v}$ or $r^{v}$ exponentiations are not randomized.

Applying additional randomization techniques on $r^{-v}$ and $r^{v}$ exponentiations could be envisaged to protect against such threats but will reduce efficiency and at the same time reason for MontExp-BRIP countermeasures.

\section{Conclusion}

Several possible implementations of BRIP algorithms have been presented in this paper. We used the efficiency of Montgomery modular arithmetic to provide an efficient message masking technique. We showed with these implementations detecting collisions through power analysis, and especially during modular multiplications, is a realistic threat. We also explained that random length must be chosen very carefully to prevent these implementations from the collision attacks we have described. Thus using 32-bit or even 64-bit random values should be avoided here. In the case where ISO random padding is used, it naturally prevents our implementation from this collision attack and allows a shorter random value (32 bits) to be used, but it is not always the case.

We also stress to the reader that random value manipulation must be strongly protected in the MontExp-BRIP algorithm against the different side channel 
techniques in order to prevent the random recovery by power leakage. Such random recovery could then lead to other classical power analysis on the secret.

\section{Acknowledgements}

The authors would like to thank Mathieu Ciet for its comments, and Sean Commercial for his help for the final version.

\section{References}

[ABDM00] Akkar, M.-L., Bevan, R., Dischamp, P., Moyart, D.: Power Analysis, What Is Now Possible. In: Okamoto, T. (ed.) ASIACRYPT 2000. LNCS, vol. 1976, pp. 489-502. Springer, Heidelberg (2000)

[AFV07] Amiel, F., Feix, B., Villegas, K.: Power Analysis for Secret Recovering and Reverse Engineering of Public Key Algorithms. In: Selected Areas in Cryptography. LNCS, vol. 4876, pp. 110-125. Springer, Heidelberg (2007)

[BCO04] Brier, E., Clavier, C., Olivier, F.: Correlation Power Analysis with a Leakage Model. In: Joye, M., Quisquater, J.-J. (eds.) CHES 2004. LNCS, vol. 3156, pp. 16-29. Springer, Heidelberg (2004)

[BK03] Bevan, R., Knudsen, E.: Ways to Enhance Differential Power Analysis. In: Lee, P.J., Lim, C.H. (eds.) ICISC 2002. LNCS, vol. 2587, pp. 327-342. Springer, Heidelberg (2003)

[CCJ04] Chevallier-Mames, B., Ciet, M., Joye, M.: Low-cost solutions for preventing simple side-channel analysis: side-channel atomicity. IEEE Transactions on Computers 53(6), 760-768 (2004)

[CF05] Ciet, M., Feix, B.: Cryptographic method comprising a modular exponentiation secured against hidden-channel attacks, crypto processor for implementing the method and associated chip card. Gemplus Patent WO2007074149 (2005)

[CG04] Ciet, M., Giraud, C.: Transient fault induction attacks on XTR. In: López, J., Qing, S., Okamoto, E. (eds.) ICICS 2004. LNCS, vol. 3269, pp. 440451. Springer, Heidelberg (2004)

[ÇKK] Koç, K.: Analysis of Sliding Window Techniques for Exponentiation. Computers and Mathematics with Applications 30(10), 17-24 (1995)

[Cor99] Coron, J.-S.: Resistance against differential power analysis for elliptic curve cryptosystems. In: Koç, Ç.K., Paar, C. (eds.) CHES 1999. LNCS, vol. 1717, pp. 292-302. Springer, Heidelberg (1999)

[DH76] Diffie, W., Hellman, M.E.: New Directions in cryptography. IEEE Transactions on Information Theory 22(6), 644-654 (1976)

[Dhe98] Dhem, J.-F.: Design of an efficient public-key cryptographic library for RISC-based smart cards. PhD thesis, Université catholique de Louvain, Louvain (1998)

[FV03] Fouque, P.-A., Valette, F.: The Doubling Attack - why upwards is better than downwards. In: Walter, C.D., Koç, Ç.K., Paar, C. (eds.) CHES 2003. LNCS, vol. 2779, pp. 269-280. Springer, Heidelberg (2003)

[Gou03] Goubin, L.: A refined power-analysis attack on elliptic curve cryptosystems. In: Desmedt, Y.G. (ed.) PKC 2003. LNCS, vol. 2567, pp. 199-210. Springer, Heidelberg (2002) 
[IIT04] Itoh, K., Izu, T., Takenaka, M.: Efficient Countermeasures against Power Analysis for Elliptic Curve Cryptosystems. In: Quisquater, J.-J., Paradinas, P., Deswarte, Y., El Kalam, A.A. (eds.) CARDIS, pp. 99-114. Kluwer, Dordrecht (2004)

[IIT06] Itoh, K., Izu, T., Takenaka, M.: Improving the Randomized Initial Point Countermeasure against DPA. In: Zhou, J., Yung, M., Bao, F. (eds.) ACNS 2006. LNCS, vol. 3989, pp. 459-469. Springer, Heidelberg (2006)

[KAK96] Koç, Ç.K., Acar, T., Kaliski, B.-S.: Analysing and comparing Montgomery multiplication algorithms. IEEE Micro. 16(3), 26-33 (1996)

$\left[\mathrm{KHK}^{+} 04\right]$ Kim, C., Ha, J., Kim, S.-H., Kim, S., Yen, S.-M., Moon, S.-J.: A Secure and Practical CRT-Based RSA to Resist Side Channel Attacks. In: Laganá, A., Gavrilova, M.L., Kumar, V., Mun, Y., Tan, C.J.K., Gervasi, O. (eds.) ICCSA 2004. LNCS, vol. 3043, pp. 150-158. Springer, Heidelberg (2004)

[KJJ99] Kocher, P.C., Jaffe, J., Jun, B.: Differential Power Analysis. In: Wiener, M.J. (ed.) CRYPTO 1999. LNCS, vol. 1666, pp. 388-397. Springer, Heidelberg (1999)

[Kob87] Koblitz, N.: Elliptic curve cryptosystems. Math. of Comp. 48(177), 203209 (1987)

[Koc96] Kocher, P.C.: Timing attacks on implementations of Diffie-Hellman, RSA, DSS, and other systems. In: Koblitz, N. (ed.) CRYPTO 1996. LNCS, vol. 1109, pp. 104-113. Springer, Heidelberg (1996)

[MDS99] Messerges, T.S., Dabbish, E.A., Sloan, R.H.: Power analysis attacks of modular exponentiation in smartcards. In: Koç, Ç.K., Paar, C. (eds.) CHES 1999. LNCS, vol. 1717, pp. 144-157. Springer, Heidelberg (1999)

[Mil86] Miller, V.S.: Use of elliptic curves in cryptography. In: Williams, H.C. (ed.) CRYPTO 1985. LNCS, vol. 218, pp. 489-502. Springer, Heidelberg (1986)

[MMM04] Mamiya, H., Miyaji, A., Morimoto, H.: Efficient Countermeasures against RPA, DPA, and SPA. In: Joye, M., Quisquater, J.-J. (eds.) CHES 2004. LNCS, vol. 3156, pp. 343-356. Springer, Heidelberg (2004)

[Mon85] Montgomery, P.L.: Modular multiplication without trial division. Mathematics of Computation 44(170), 519-521 (1985)

[MOV96] Menezes, A., van Oorschot, P.C., Vanstone, S.A.: Handbook of Applied Cryptography. CRC Press, Boca Raton (1996)

[MPO05] Mangard, S., Pramstaller, N., Oswald, E.: Successfully attacking masked AES hardware implementations. In: Rao, J.R., Sunar, B. (eds.) CHES 2005. LNCS, vol. 3659, pp. 157-171. Springer, Heidelberg (2005)

[RSA78] Rivest, R.L., Shamir, A., Adleman, L.: A method for obtaining digital signatures and public-key cryptosystems. Communications of the ACM 21, 120-126 (1978)

[YLMH05] Yen, S.-M., Lien, W.-C., Moon, S., Ha, J.: Power Analysis by Exploiting Chosen Message and Internal Collisions - Vulnerability of Checking Mechanism for RSA-decryption. In: Dawson, E., Vaudenay, S. (eds.) Mycrypt 2005. LNCS, vol. 3715, pp. 183-1956. Springer, Heidelberg (2005) 


\section{A Montgomery Arithmetic}

\section{A.1 Montgomery Multiplication}

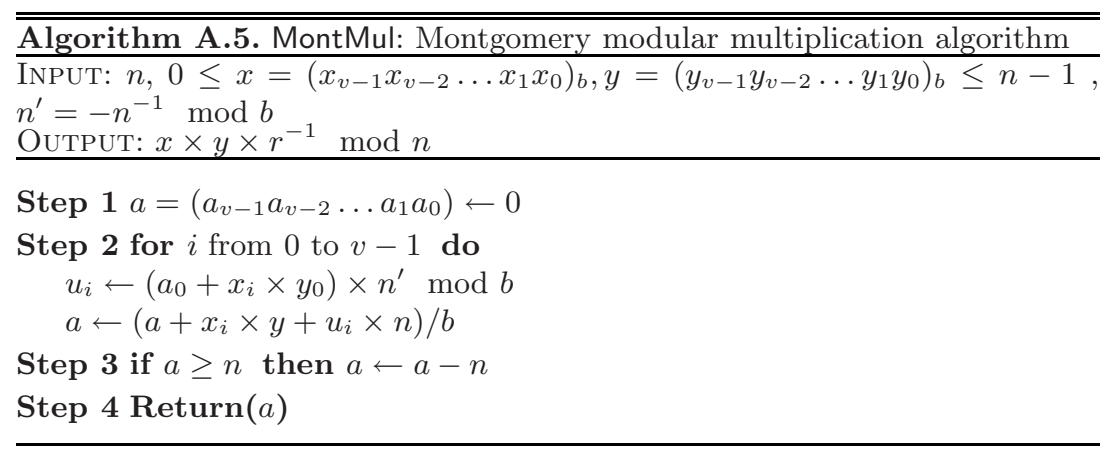

\section{A.2 Montgomery Exponentiation}

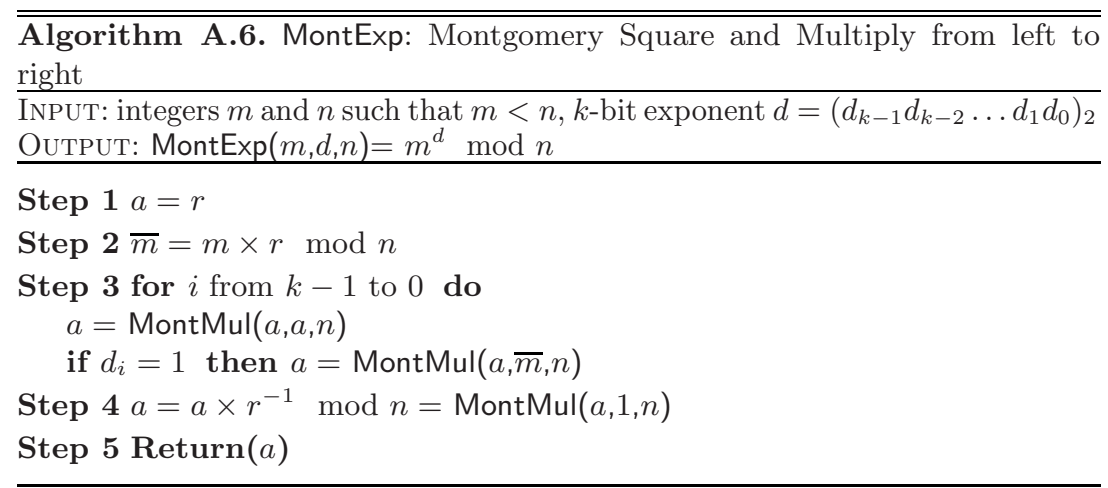

